ReVISTA de BIOLOGía TROPICAL

\title{
Tolerancia de diez especies de diatomeas (Bacillariophyceae) a los factores físico-químicos del agua en el Río Sarapiquí, Costa Rica
}

\author{
Edwin Céspedes-Vargas ${ }^{1,2}$, Gerardo Umaña-Villalobos ${ }^{2,3}$ \& Ana Margarita Silva-Benavides ${ }^{2,3}$ \\ 1. 922 - 4050 Alajuela, Costa Rica; edwincespedesv@gmail.com \\ 2. Escuela de Biología, Universidad de Costa Rica, San Pedro, San José 11501, Costa Rica; \\ gerardo.umana@ucr.ac.cr, ana.silva@ucr.ac.cr \\ 3. Centro de Investigación en Ciencias del Mar y Limnología (CIMAR), Universidad de Costa Rica, San Pedro, San José \\ 11501, Costa Rica.
}

Recibido 10-III-2015. C Corregido 22-VIII-2015. Aceptado 24-IX-2015.

\begin{abstract}
The tolerance of ten diatom species (Bacillariophyceae) to water's physico-chemical factors in the Sarapiqui River, Costa Rica. Benthic diatoms are the periphyton component most studied in Costa Rican rivers, yet there is still much to be known about their ecology. This study aims to contribute to this knowledge. Periphyton samples from six locations along the middle reach of Sarapiquí river and some of its tributaries were taken and analyzed from 2010 to 2012. A total count of 400 frustules was performed in each sample to obtain relative abundances of each species. Ten species, which were easily recognized at the light microscope, were analyzed here: Achnanthidium exiguum, Coconneis placentula, Cymbella tumida, Luticola goeppertiana, Luticola ventricosa, Navicula symmetrica, Nitzchia clausii, Nupela praecipua, Reimeria sinuata and Synedra goulardi. Their abundance was related to physico chemical data at each site for every sampling date (dissolved oxygen, temperature, conductivity, total suspended solids, turbidity, $\mathrm{pH}$ and alkalinity) with a canonical correspondence analysis (CCA) that allowed distinguishing three sectors along the river according to altitude. These sectors differed by substrate, riparian vegetation cover, and physic-chemical parameters, all of which resulted in differences in diatom species composition among them. Optimum values and tolerances form each environmental parameter were calculated for each species. Our results showed that A. exiguum, R. sinuata, $L$. ventricosa and $C$. tumida, were dominant in clean and well oxygenated waters with low temperatures. The species $N$. praecipua, S. goulardi, C. placentula, L. goeppertiana, N. clausii and N. symmetrica inhabited warm waters with high turbidity and low oxygen. The species $A$. exiguum, $R$. sinuata were dominant in sites with low alkalinity and conductivity, whereas species like $N$. symmetrica, C. tumida and L. ventricosa showed a positive relationship with conductivity and $\mathrm{pH}$. The studied species showed good characteristics of their value as water quality indicators, yet due to different interpretations by several authors, it is necessary to perform further studies at more river basins in the country. Rev. Biol. Trop. 64 (1): 105-115. Epub 2016 March 01.
\end{abstract}

Key words: Costa Rica, diatoms, ecology, water physico chemistry, Sarapiquí River, tolerance, optimum values.

La ecología de los ríos y riachuelos fue inicialmente resumida por Hynes (1970), quien los describe como ambientes donde predominan organismos del bentos, cuyos hábitos de vida se asocian al material del fondo y la composición de la comunidad depende de la estructura física del material del sustrato. En los ríos de montaña y zonas bajas el sustrato normalmente consiste de grandes rocas o cantos rodados (Leopold, Wolman, \& Miller, 1992) sobre los que crece el perifiton como productor primario principal. El perifiton es una comunidad compleja que incluye varios grupos de algas, hongos, bacterias y protozoos o micro metazoos (Azim, Beveridge, van Dam, \& Verdegem, 2005; Roldán-Pérez \& RamírezRestrepo, 2010). Uno de los componentes más importantes son las diatomeas (Clase Bacillariophyceae, División Heterokontophyta) (Lee, 2008). Las diatomeas son reconocidas mundialmente como indicadoras de contaminación orgánica y eutrofización (Lobo et al., 
2010), pues como grupo poseen una amplia distribución, permitiendo la comparación en diversos habitats. Son excelentes indicadores por su alta tasa reproductiva y presentan una respuesta rápida a los cambios de las condiciones de los cuerpos del agua (Schneck, Torgan, \& Schwarzbold, 2007), y una pared de sílice resistente a los daños causados por la remoción a partir de un substrato rígido.

En el continente americano, las diatomeas han sido investigadas desde el punto de vista taxonómico (Metzeltin \& Lange-Bertalot, 1998; 2007; Ponader \& Potapova, 2007; Sala, Ramírez, \& Plata, 2008; Vouilloud, Sala, Nunez, \& Duque, 2010) y como bioindicadoras (Potapova \& Charles, 2007; Bere \& Tundisi, 2010; 2011a; 2011b; Tudesque, Grenuillet, Gevrey, Khazraie, \& Brosse, 2012). En Costa Rica, se ha estudiado el uso de las diatomeas como indicadoras de diferentes condiciones ambientales (Silva-Benavides, 1996b; Michels, 1996, 1998a; 1998b; Michels, Umaña, \& Raede, 2006; Nunn, 1999). Otros trabajos se han enfocado en las diatomeas desde un punto de vista ecológico y descriptivo de las especies (Phillips, 1987; Silva, 1994; SilvaBenavides, 1996a; Michels, 1996; Wydrzycka \& Lange-Bertalot, 2001; Michels-Estrada, 2003; Silva-Benavides, Sili, \& Torzillo, 2008). Los resultados han demostrado que la falta de conocimiento en especies de diatomeas tropicales, genera un problema al procurar aplicar los índices de biomonitoreo desarrollados en Europa (Michels, 1996; 1998a; 1998b).

El monitoreo biológico en ríos es importante debido a que los organismos presentan una respuesta integral a las fluctuaciones del ambiente acuático, y con ello brindan una mejor noción de la calidad del agua en las que fueron encontradas, que las variables físico químicas, que dan valores puntuales. Por lo tanto, si se desea mantener la salud y la diversidad de las comunidades biológicas, monitorear la comunidad acuática da información única y muy valiosa. Sin embargo, la escogencia de un grupo bioindicador debe satisfacer ciertos criterios, como que cada taxón posea límites ecológicos definidos y que el taxón pueda ser identificado sin equivocación (Cox, 1991). Por este motivo, el objetivo del presente trabajo consistió en evaluar la tolerancia a los factores físico-químicos del agua de diez especies de diatomeas (Bacillariophyceae) en el Río Sarapiquí, Costa Rica. Con esto se espera contribuir al conocimiento de la ecología de las diatomeas y al biomonitoreo de un río de un país tropical, como Costa Rica.

\section{MATERIALES Y MÉTODOS}

Sitios de muestreo: El Río Sarapiquí nace en el costado oeste de las faldas del volcán Barva, Costa Rica a una altura aproximada de $1650 \mathrm{~m}$ y corre dirección Norte-Noreste, hasta desembocar en el Río San Juan a 17 m de elevación. Los sitios muestreados se encuentran desde los $180 \mathrm{~m}$ a $700 \mathrm{~m}$ de elevación, lo que equivale a la cuenca media-baja (Apéndice 1). La cuenca ha sido explotada con fines agrícolas, especialmente en la margen izquierda de la parte media a baja del Río Sarapiquí, lo que ha degradado los ecosistemas naturales.

Se localizaron seis estaciones de muestreo: tres en el cauce del Río Sarapiquí: el sitio Colonia del Socorro (Sar. 1) se localizó a 700 m de elevación $\left(10^{\circ} 15^{\prime} 27^{\prime \prime} \mathrm{N}-84^{\circ} 10^{\prime} 13^{\prime \prime}\right.$ W); el segundo sitio San Miguel (Sar. 2) a 380 m de elevación (10¹9'38' N - 84¹0'14" W) y el tercero en la entrada a la Reserva La Tirimbina en la Virgen de Sarapiquí (Sar. 3) a 162 m de elevación $\left(10^{\circ} 24^{\prime} 56^{\prime \prime} \mathrm{N}-84^{\circ} 7^{\prime} 19^{\prime}\right.$ ' W). Los otros tres sitios fueron ubicados en afluentes los cuales corresponden al Río Volcán (Vol) a $380 \mathrm{~m}$ de elevación (10¹9’31" N - 84¹0'4" W); Río San Ramón (S. Ram) a $200 \mathrm{~m}$ de elevación $\left(10^{\circ} 23^{\prime} 47^{\prime}\right.$ ' $\mathrm{N}-84^{\circ} 7^{\prime} 28^{\prime}$ ' W) y Río Tirimbina (Tiri) a $180 \mathrm{~m}$ de elevación $\left(10^{\circ} 23\right.$ '60" N - 846'23" W).

El área de estudio presenta un promedio de precipitación anual de 4000 a $5000 \mathrm{~mm}$, con un periodo de lluvias que varía de 250 a 300 días. La temperatura oscila anualmente entre los $14-26{ }^{\circ} \mathrm{C}$. El promedio anual de brillo solar es de $5 \mathrm{a}<3 \mathrm{~h}$ al día y presenta una evaporación anual que oscila entre los $1000 \mathrm{~mm}$ a $1200 \mathrm{~mm}$ anuales (IMN, 2009). Los sitios 
se encuentran en las zonas de vida de Pluvial premontano (Sar.1), pasando por Muy húmedo tropical, transición a premontano (Sar. 2 y Vol) a Muy húmedo tropical (Sar. 3, Tiri y S. Ram).

El sustrato de los sitios dentro del cauce principal del Río Sarapiquí fue principalmente pedregoso arenoso, con la presencia de grandes cantos rodados. El sitio Volcán presentó características similares al sitio San Miguel, con un sustrato pedregoso arenoso y la presencia de grandes rocas. Además, todos estos sitios carecieron de una extensa cobertura boscosa, ya que el cauce de los ríos es ancho, característica que evita que la vegetación se establezca en sus orillas. El sustrato en los sitios de los ríos San Ramón y Tirimbina fue principalmente de sedimentos finos con la presencia de pequeñas rocas, además presentó una vasta cobertura boscosa en su zona riparia.

Recolecta y preparación de las muestras: Las recolectas se realizaron los días 1 y 2 de octubre 2010; 8 y 9 de abril 2011; 28 y 29 de julio 2011; 11y 12 de noviembre 2011; 1 y 2 de junio 2012. Las muestras se obtuvieron mediante el raspado con un cepillo dental de la superficie de las rocas (Michels-Estrada, 2003), las cuales se extrajeron en un transecto perpendicular al río, desde la orilla hasta la zona más profunda. Se conservaron con formalina al 10 $\%$ para su transporte al laboratorio del CIMAR, Universidad de Costa Rica.

Para la limpieza de las diatomeas se realizó una digestión con ácido clorhídrico concentrado y con peróxido de hidrógeno (CéspedesVargas, 2014). Cada muestra fue fijada a un portaobjetos con resina Permount ${ }^{\circledR}$ y cubreobjetos. Las diatomeas fueron identificadas taxonómicamente a nivel de especie y cuantificadas con un microscopio de luz Olympus modelo IX-51, con lente de aceite de inmersión a un aumento de $1000 \mathrm{x}$. Con el propósito de corroborar la identificación realizada en el microscopio de luz de las 10 especies más abundantes, se procedió a la observación con el microscopio electrónico de barrido Zeiss modelo DSM 942. Previamente, cada muestra fue deshidratada a temperatura ambiente sobre bases metálicas y posteriormente cubiertas con una capa de oro (Hasle \& Fryxell, 1970).

Variables fisico-químicas del agua: El oxígeno disuelto y la temperatura fueron medidos con un oxímetro YSI550A. La conductividad fue registrada con un conductímetro Lamotte Chemical. El total de sedimentos en suspensión (TSS) fueron calculados por el método gravimétrico (APHA, 2005). La turbidez del agua en los sitios fue medida con un equipo 2020 marca Lamotte. El pH se midió con tiras indicadoras de $\mathrm{pH}$, Indicador Universal marca Merck. La alcalinidad se calculó con un equipo de alcalinidad marca Hach.

Con el propósito de determinar la frecuencia relativa de las especies se realizó un conteo de 400 frústulas por cada lámina (Lowe \& LaLiberté, 2006). Para el análisis se consideraron las 10 especies más frecuentes cuantificadas e identificadas en el microscopio de luz y corroboradas al microscopio electrónico. Con estos datos se cuantificaron las abundancias relativas de cada especie.

Para definir las variables físico-químicas del agua que influyen en la abundancia de las especies se realizó un Análisis de Correspondencia Canónica (CCA) (Legendre \& Legendre, 1998) con el programa estadístico PAST3 (Hammer, Harper \& Ryan, 2001). Los valores de estas variables fueron estandarizadas para su posterior análisis.

Se calcularon los valores óptimos y la tolerancia de las especies para las variables ambientales por medio del método del promedio ponderado. Se ponderó el valor de cada variable ambiental con base en la abundancia relativa de la especie en los sitios muestreados (Leps \& Smilauer, 2003).

\section{RESULTADOS}

Las especies para las cuales fue posible realizar el análisis de asociación con los factores físicoquímicos estudiados fueron: Achnanthidium exiguum (Grunow) Czarnecki, Coconneis placentula Ehrenberg, Cymbella tumida (Brébisson) van Heurck, Luticola 
goeppertiana (Bleisch) D. G. Mann, Luticola ventricosa (Kützing) D. G. Mann, Navicula symmetrica Patrick, Nitzchia clausii Hantzsch, Nupela praecipua (E. Reichardt) E. Reichardt, Reimeria sinuata (Gregory) Kociolek \& Stoermer y Synedra goulardi Brébisson ex Cleve \& Grunow. Los valores promedio y desviación estándar de los parámetros físico-químicos se presentan en el cuadro 1.

Por medio del Análisis de Correspondencia Canónica (CCA) se obtuvo la figura 1. Los ejes 1 y 2 explicaron en conjunto un $58.18 \%$ de la abundancia de las 10 especies. El primer eje de ordenación se relacionó principalmente con

\section{CUADRO 1}

Valores promedio y desviación estándar de los parámetros físico químicos de los sitios estudiados en la cuenca del Río Sarapiquí, Costa Rica

TABLE 1

Mean values and their standard deviation of physic chemical parameters at the sampled sites in the drainage of River Sarapiquí, Costa Rica

\begin{tabular}{lccccccc}
\multicolumn{1}{c}{ Sitio } & O.D. $(\mathrm{mg} / \mathrm{L})$ & $\mathrm{pH}$ & $\begin{array}{c}\text { Alcalinidad } \\
(\mathrm{mg} / \mathrm{L})\end{array}$ & $\begin{array}{c}\text { Conductividad } \\
(\mu \mathrm{Si} / \mathrm{cm})\end{array}$ & $\begin{array}{c}\text { Temperatura } \\
\left({ }^{\circ} \mathrm{C}\right)\end{array}$ & $\begin{array}{c}\text { Turbidez } \\
(\mathrm{NTU})\end{array}$ & $\begin{array}{c}\text { TSS } \\
(\mathrm{mg} / \mathrm{L})\end{array}$ \\
Sar. 1 & $8.8 \pm 0.6$ & $8.0 \pm 0.9$ & $42.7 \pm 6.2$ & $99.3 \pm 38.4$ & $19.8 \pm 1.2$ & $2.7 \pm 1.8$ & $6.2 \pm 4.0$ \\
Sar. 2 & $8.9 \pm 1.01$ & $8.5 \pm 0.5$ & $21.9 \pm 7.4$ & $138.6 \pm 37.0$ & $21.4 \pm 1.3$ & $6.6 \pm 3.3$ & $10.5 \pm 9.3$ \\
Sar. 3 & $8.7 \pm 0.5$ & $7.8 \pm 0.6$ & $43.2 \pm 9.1$ & $86.9 \pm 16.6$ & $22.5 \pm 1.1$ & $8.5 \pm 9.9$ & $15.6 \pm 18.4$ \\
Vol & $9.4 \pm 0.8$ & $7.6 \pm 0.9$ & $20.3 \pm 5.5$ & $58.4 \pm 16.6$ & $21.1 \pm 1.3$ & $1.4 \pm 1.6$ & $2.2 \pm 1.1$ \\
Tiri & $8.3 \pm 0.4$ & $7.2 \pm 0.4$ & $27.6 \pm 3.9$ & $73.3 \pm 25.8$ & $23.5 \pm 1.6$ & $2.5 \pm 2.3$ & $1.7 \pm 1.5$ \\
S. Ram & $8.5 \pm 0.4$ & $7.6 \pm 0.8$ & $33.9 \pm 8.7$ & $87.5 \pm 14.3$ & $23.7 \pm 0.6$ & $4.0 \pm 3.8$ & $10.5 \pm 16.2$ \\
\hline
\end{tabular}

Nombres de los sitios según se explica en materiales y métodos.

Site names are explained at the materials and methods section.

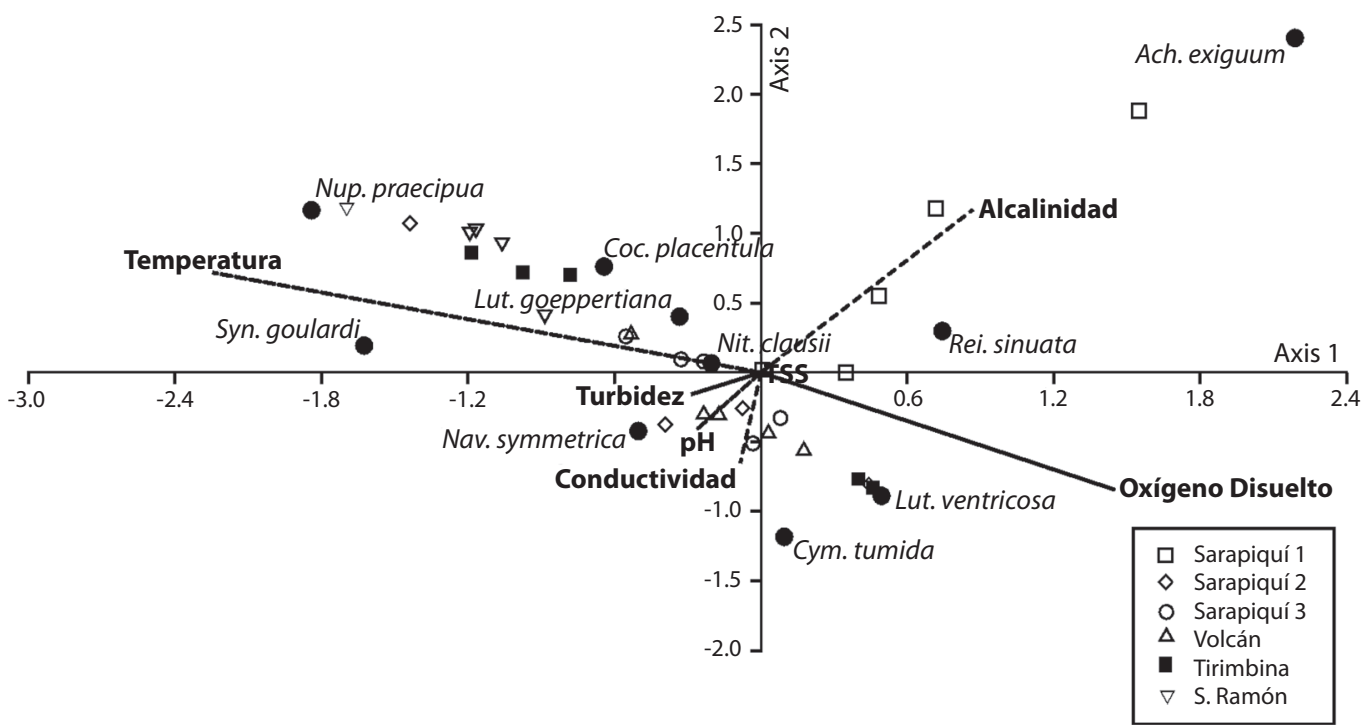

Fig. 1. Análisis de Correspondencia Canónica que muestra la distribución de las especies por sitio y su relación con los parámetros físico químicos. Ambos ejes explican un $58 \%$ de la variancia total. Río Sarapiquí, Costa Rica.

Fig. 1. Canonical Correspondence Analysis showing the species distribution per site and their relationship with physic chemical parameters. Both axis explain $58 \%$ of total variance. Río Sarapiquí, Costa Rica. 
la temperatura, el oxígeno disuelto y la turbidez, separando las muestras de los ríos S. Ram y Tiri, de las muestras de Sar.1. Las muestras provenientes de los sitios Vol, Sar.2 y Sar.3 fueron agrupadas en la parte media del gráfico. Las especies $A$. exiguum, $R$. sinuata, $L$. ventricosa y $C$. tumida, se relacionaron con aguas de baja temperatura, bien oxigenadas y baja turbidez. El segundo grupo de especies $N$. praecipua, $S$. goulardi, C. placentula, L. goeppertiana, $N$. clausii y N. symmetrica, a la izquierda del gráfico, se relacionaron principalmente con aguas cálidas poco oxigenadas y alta turbidez. Para la especie $N$. symmetrica se observó una fuerte relación positiva con la turbidez.

El segundo eje se relacionó principalmente con la alcalinidad, la conductividad, el $\mathrm{pH}$ y el total de sólidos en suspensión. Las especies $A$. exiguum y $R$. sinuata, se relacionaron con aguas alcalinas, baja conductividad y bajos niveles de $\mathrm{pH}$. Las especies L. goeppertiana, $N$. clausii y $C$. placentula presentaron una relación positiva con la cantidad de TSS. Las especies $N$. symmetrica, C. tumida y L. ventricosa presentaron una relación positiva con la conductividad y el $\mathrm{pH}$ de las aguas.

Los resultados del análisis de correspondencia canónica permitieron entender las diferencias entre los sitios con base en sus características físico-químicas, su ubicación y su composición florística. El sitio Sar.1 se ubicó en la parte más alta de la cuenca media, con aguas limpias y bien oxigenadas, donde dominaron las especies $A$. exiguum y $R$. sinuata. Los sitios Sar. 2, Sar. 3 y Vol se ubicaron en la zona intermedia del gráfico del CCA (Fig. 1), similares en términos de cobertura boscosa y tipo de sustrato, representados por las especies $N$. clausii y $N$. symetrica . Los sitios Tiri y S. Ram, aunque presentaron una amplia cobertura boscosa riparia que impide la penetración de los rayos solares la mayor parte del día, fueron los sitios de mayor temperatura, donde prevalecieron las especies N. praecipua, C. placentula, S. goulardi y L. goeppertiana.

Los resultados obtenidos para los óptimos y la tolerancia de las especies relacionados con las diferentes variables fisicoquímicas se resumen en el cuadro 2 . Respecto al oxígeno disuelto, las especies presentaron niveles óptimos relativamente altos, donde la especie $C$. tumida presentó una mayor tolerancia al cambio de oxígeno disuelto. C. tumida fue la especie que presentó una mayor tolerancia al cambio de oxígeno disuelto. Las especies presentaron tolerancias similares con un nivel óptimo de $\mathrm{pH}$ mayor a 7 . La especie L. goeppertiana fue la que presentó una mayor tolerancia a los

\section{CUADRO 2}

Valores en la cuenca media del Río Sarapiquí, Costa Rica

TABLE 2

Optimal values and tolerance $( \pm)$ for the ten diatom species included in the study at the middle reaches of the drainage basin of River Sarapiquí, Costa Rica

\begin{tabular}{lccccccc}
\multicolumn{1}{c}{ Especie } & $\begin{array}{c}\text { Temperatura } \\
\left({ }^{\circ} \mathrm{C}\right)\end{array}$ & $\begin{array}{c}\mathrm{OD} \\
(\mathrm{mg} / \mathrm{L})\end{array}$ & $\mathrm{pH}$ & $\begin{array}{c}\text { Alcalinidad } \\
(\mathrm{mg} / \mathrm{L})\end{array}$ & $\begin{array}{c}\text { Conductividad } \\
(\mu \mathrm{S} / \mathrm{cm})\end{array}$ & $\begin{array}{c}\text { Turbidez } \\
\text { NTU }\end{array}$ & $\begin{array}{c}\text { TSS } \\
(\mathrm{mg} / \mathrm{L})\end{array}$ \\
Achnantidium exiguum & $20.6 \pm 1.4$ & $8.9 \pm 0.6$ & $7.4 \pm 0.6$ & $43.9 \pm 6.9$ & $79.0 \pm 21.9$ & $3.6 \pm 2.0$ & $8.7 \pm 3.9$ \\
Coconneis placentula & $22.4 \pm 2.1$ & $8.6 \pm 0.6$ & $7.7 \pm 0.7$ & $33.4 \pm 10.2$ & $81.8 \pm 25.9$ & $4.1 \pm 5.8$ & $7.8 \pm 11.8$ \\
Cymbella tumida & $21.2 \pm 1.4$ & $9.1 \pm 0.9$ & $7.8 \pm 0.7$ & $24.9 \pm 9.0$ & $79.2 \pm 30.4$ & $2.8 \pm 5.2$ & $4.6 \pm 9.0$ \\
Luticola goeppertiana & $21.2 \pm 2.3$ & $8.6 \pm 0.5$ & $8.2 \pm 0.8$ & $30.0 \pm 6.9$ & $79.1 \pm 28.6$ & $4.0 \pm 2.7$ & $5.0 \pm 6.8$ \\
Luticola ventricosa & $21.5 \pm 1.1$ & $9.1 \pm 0.8$ & $7.5 \pm 0.7$ & $30.1 \pm 4.9$ & $92.2 \pm 23.6$ & $4.7 \pm 5.8$ & $9.9 \pm 12.1$ \\
Navicula symmetrica & $21.4 \pm 1.2$ & $8.5 \pm 0.5$ & $8.2 \pm 0.7$ & $30.4 \pm 10.3$ & $124.1 \pm 44.0$ & $5.3 \pm 6.8$ & $8.8 \pm 12.8$ \\
Nitzschia clausii & $22.0 \pm 1.0$ & $8.9 \pm 0.6$ & $7.7 \pm 0.6$ & $33.1 \pm 7.4$ & $108.0 \pm 29.9$ & $6.3 \pm 4.7$ & $9.0 \pm 8.8$ \\
Nupela praecipua & $23.2 \pm 1.3$ & $8.5 \pm 0.4$ & $7.9 \pm 0.6$ & $31.5 \pm 6.8$ & $85.7 \pm 17.7$ & $6.3 \pm 3.2$ & $7.9 \pm 10.1$ \\
Reimeria sinuata & $20.6 \pm 1.5$ & $8.6 \pm 0.5$ & $8.0 \pm 0.8$ & $34.1 \pm 11.0$ & $104.27 \pm 35.00$ & $3.6 \pm 2.6$ & $5.9 \pm 4.4$ \\
Synedra goulardi & $23.2 \pm 1.5$ & $8.4 \pm 0.6$ & $7.7 \pm 0.7$ & $27.8 \pm 6.7$ & $81.94 \pm 32.19$ & $5.1 \pm 6.1$ & $8.1 \pm 14.1$ \\
\hline
\end{tabular}


cambios en el $\mathrm{pH}$. Con respecto a la alcalinidad, la mayoría de las especies mostraron niveles óptimos cercanos a los $30 \mathrm{mg} / \mathrm{L}$, A. exiguum presentó el valor óptimo más alto, mientras que las especies $C$. placentula, $N$. symmetrica y $R$. sinuata presentaron una amplia tolerancia a los cambios de alcalinidad.

Los valores óptimos de conductividad fueron mayores en $N$. symmetrica, $N$. clausii y $R$. sinuata. Además $N$. symmetrica presentó una alta tolerancia a cambios de este parámetro. Los valores óptimos de temperatura para las diez especies se encontraron superiores a $20^{\circ}$ C y C. placentula y L. goeppertiana fueron las especies que presentaron ámbitos más amplios de tolerancia.

Las especies $N$. clausii y $N$. praecipua presentaron los dos valores óptimos más altos de tubidez, seguidos por $N$. symmetrica y $S$. goulardi. Estas últimas fueron las que mostraron una mayor tolerancia a los cambios en la turbidez. Otras especies como C. placentula, C. tumida y L. ventricosa también presentaron niveles altos de tolerancia a la turbidez.

Las especies $L$. ventricosa y $N$. clausi obtuvieron los valores óptimos más altos respecto al TSS, seguidas por $N$. symmetrica y $A$. exiguum, respectivamente. Sin embargo, fue la especie $S$. goulardi la que mostró la mayor tolerancia a la variación de los sólidos en suspensión. Otras especies como L. ventricosa y $N$. symmetrica también presentaron ámbitos de tolerancia amplios.

\section{DISCUSIÓN}

Según los resultados obtenidos en esta investigación, A. exiguum prefiere aguas oxigenadas y alcalinas, datos que concuerdan con los resultados obtenidos por Nunn (1999) y Morales, et al. (2011). Además presentó un valor óptimo alto respecto al TSS dato que concuerda con Silva-Benavides (1996b), quien la clasificó como una especie tolerante a una alta contaminación orgánica. La especie C. placentula se relacionó principalmente con aguas cálidas, poco oxigenadas y alta turbidez, que la clasifica como una especie altamente tolerante a la contaminación. La especie se ha reportado en ambientes con una fuerte contaminación con materia orgánica y clasificada como una especie eutrófica (Silva-Benavides, 1996a). La especie presenta una respuesta negativa a la exposición lumínica (Goldsborough, 1994; Michels-Estrada, 2003), que se demostró en su mayor abundancia en sitios con una alta cobertura vegetal en el presente trabajo.

Cymbella tumida, la cual es considerada por Krammer (2002) como una especie de distribución exclusivamente tropical, se relacionó principalmente con aguas bien oxigenadas y baja temperatura en el presente trabajo, observaciones que concuerdan con Nunn (1999) y Silva-Benavides (1996a). Otros investigadores han notado que la especie es intolerante a la contaminación (Silva-Benavides, 1996a; Michel, 1998a), presenta una predilección por aguas oligotróficas a mesotróficas con una cantidad media de electrolitos (Krammer, 2002), lo que podría explicar su relación positiva con la conductividad y el $\mathrm{pH}$ principalmente en los sitios Sar. 1 y Vol.

La especie L. goeppertiana mostró un amplio ámbito de tolerancia a cambios en la temperatura y $\mathrm{pH}$, presentándose principalmente en los sitios con aguas cálidas y alta turbidez, lo cual suponemos le brinda sus cualidades como una especie tolerante a la contaminación. Se encuentra relacionada con los sitios que presentaron una menor cobertura boscosa (Sar. 2, Vol), lo que concuerda con los datos obtenidos con Michels-Estrada (2003), quien señala que es una especie común en aguas lóticas con poca sombra y con una alta concentración de partículas en suspensión. Esta especie ha sido catalogada como buena indicadora de aguas fuertemente contaminadas con desechos orgánicos (Silva-Benavides, 1996b), relacionados a sitios con alto impacto antropogénico (Schneck et al., 2007).

La especie del mismo género L. ventricosa presenta una menor tolerancia a la contaminación que L. goeppertiana (Krammer \& LangeBertalot, 1986). La especie es abundante en los sitios con aguas de baja temperatura, turbidez y bien oxigenadas como Sar. 1, Sar. 2 y 
Vol. Silva-Benavides (1996b) la cataloga como sensible a la contaminación, sin embargo, en el presente trabajo se observó que la especie presenta una tolerancia alta a los cambios en la turbidez y el TSS.

La distribución de $N$. symmetrica fue mayor en los sitios que presentaron aguas cálidas poco oxigenadas y con alta turbidez como Sar. 2; mostró además una predilección por ambientes con alta conductividad y $\mathrm{pH}$. Los resultados obtenidos en esta investigación concuerdan en parte con los resultados de Michels et al. (2006), donde la especie se relaciona positivamente con la turbidez y el $\mathrm{pH}$, pero negativamente con respecto a la sombra.

La especie $N$. clausii presentó un comportamiento similar a L. goeppertiana. Se reporta en aguas salobres, cerca de las costas y estuarios de ríos que presentan altas concentraciones de electrolitos (Krammer \& Lange-Bertalot, 1988). Michels et al. (2006), reportaron una abundancia significativa de individuos de esta especie en ambientes saturados de sedimentos y alta demanda bioquímica de oxígeno (DBO). Estas observaciones concuerdan con nuestros resultados donde prevalece una relación positiva entre el número de individuos y el aumento de TSS. Silva-Benavides (1996a), la cataloga como una especie frecuente en aguas oxigenadas y contaminadas con materia orgánica. Los altos valores óptimos que presenta para la conductividad y la turbidez, así como el amplio ámbito de tolerancia a cambios en el TSS, le brindan una mayor capacidad de adaptación a la contaminación de las aguas.

La especie de distribución tropical $N$. praecipua, (Michels, 1998b; Nunn, 1999), se relaciona de acuerdo con nuestro estudio con aguas cálidas, poco oxigenadas y alta turbidez; pero no es tolerante a la alcalinidad del agua. $\mathrm{Su}$ alta presencia en sitios sombreados concuerda con Michels et al. (2006), quien la describe como una especie que prefiere sitios con baja luminosidad. Su alta abundancia en los sitios cuya agua presentó alta turbidez se relaciona con las observaciones de Schneck et al. (2007), quienes la clasifican como una especie indicadora de aguas contaminadas. Sin embargo, sugieren que a pesar de su alta tolerancia a la contaminación, puede aparecer también en aguas limpias. Relacionado con este último concepto, Wydrzycka y Lange-Bertalot (2001) y Michels-Estrada (2003) consideran esta especie como indicadora de aguas limpias y bien oxigenadas. Por tratarse de una especie de distribución tropical se deberían aunar esfuerzos para recopilar mayor información sobre la ecología de esta especie, la cual fue muy frecuente en casi todas las muestras del presente estudio y podría ser una excelente herramienta en la bioindicación de la calidad en ríos limpios en el bosque lluvioso tropical.

En el presente trabajo $R$. sinuata se localizó en el grupo de especies que presentaron una predilección por aguas de baja temperatura, bien oxigenadas y baja turbidez. De acuerdo con Levkov y Ector (2010) es una de las diatomeas más frecuentes en ecosistemas de agua dulce, tolerante a altos niveles de eutrofización y alcalinos (Veselá \& Johansen 2009; O’Driscoll et al., 2012). Inicialmente se clasificó como una especie de zonas altas (Krammer \& Lange-Bertalot, 1986), sin embargo, se ha encontrado también en ambientes de poca elevación (Morales et al., 2007). Por su amplia distribución, coincidimos con Silva-Benavides (1996b) de catalogarla como una mala indicadora de calidad de agua.

S. goulardi se presume esta confinada a las zonas tropicales de Norte y Sur América, en Centroamérica y el trópico en África (Williams, 1986; Silva-Benavides, 1996b; Michels, 1998b). Esta especie presentó preferencias ecológicas similares a $N$. praecipua: aguas cálidas y poco oxigenadas. Estos resultados coinciden con los obtenidos por Krammer y Lange-Bertalot (1991), Nunn (1999) y Silva-Benavides et al. (2008), quienes reportan esta especie común en aguas cálidas no contaminadas. De acuerdo a nuestras observaciones podemos afirmar que $S$. goulardi prefiere los ambientes de aguas cálidas. La información ecológica de esta especie es escasa en la literatura, por lo tanto, al igual que con N. praecipua, se debería investigar más sobre su ecología. 
Se pueden distinguir en la cuenca media del R. Sarapiquí tres sectores de acuerdo a la altitud, donde cada sector se caracteriza por poseer propiedades únicas en lo que se refiere al tipo de sustrato, cobertura riparia vegetal y diferentes parámetros físico-químicos, características que propician diferencias en la composición de especies de diatomeas entre los sectores. Las especies A. exiguum, $R$. sinuata, $L$. ventricosa y $C$. tumida, se relacionan con aguas de baja temperatura, bien oxigenadas y baja turbidez. Las especies $N$. praecipua, $S$. goulardi, C. placentula, L. goeppertiana, $N$. clausii y $N$. symmetrica se relacionan principalmente con aguas cálidas poco oxigenadas y alta turbidez. Además, las especies $A$. exiguum, $R$. sinuata, se relacionan con aguas de alta alcalinidad, baja conductividad y niveles de $\mathrm{pH}$ cercanos a 7. Las especies $L$. goeppertiana, $N$. clausii y $C$. placentula presentan una relación positiva con la cantidad de TSS. Las especies $N$. symmetrica, $C$. tumida y $L$. ventricosa presentan una relación positiva con la conductividad y el $\mathrm{pH}$ de las aguas. Las especies de diatomeas estudiadas ofrecen indicios importantes sobre la calidad del agua del río, lo que constituye una herramienta importante para la investigación y monitoreo de un ecosistema tropical. Sin embargo, debido a las diversas interpretaciones realizadas por diferentes autores sobre una misma especie y en algunos casos a dificultades de tipo taxonómico, se requiere de un análisis individualizado para cada región tal y como se ha hecho en el presente estudio.

\section{AGRADECIMIENTOS}

El trabajo se realizó en el Centro de Investigación en Ciencias del Mar y Limnología, Universidad de Costa Rica dentro del proyecto 808-A9-230 financiado por la Vicerrectoría de Investigación de la Universidad de Costa Rica. Se agradece la colaboración de Steven Jansen, Universidad de Ulm por proporcionar el microscopio electrónico. Ellen Salzer y Hans Malchus por su asistencia técnica en el procesamiento y análisis de las muestras en microscopia electrónica. Aldo Farah colaboró con el análisis de las muestras de agua y Jeffry Sibaja colaboró con el análisis estadístico de los datos.

\section{RESUMEN}

Las diatomeas del bentos son el componente más estudiado del perifiton en los ríos de Costa Rica, sin embargo aún queda mucho por conocer sobre su ecología. Por eso en el presente estudio se analizó el perifiton en seis diferentes sitios en la cuenca medio del Río Sarapiquí en el 2010, 2011 y 2012. En cada uno de los muestreos se realizó el conteo de 400 frústulas, de las cuales se obtuvo la abundancia relativa de las especies: Achnanthidium exiguum, Coconneis placentula, Cymbella tumida, Luticola goeppertiana, Luticola ventricosa, Navicula symmetrica, Nitzchia clausii, Nupela praecipua, Reimeria sinuata y Synedra goulardi. Esta información fue relacionada con los datos físico químicos (oxígeno disuelto, temperatura, conductividad, total de sedimentos en suspensión, turbidez, $\mathrm{pH}$ y alcalinidad) medidos en cada uno de los sitios. Con el propósito de observar las relaciones entre los diferentes sitios dentro de la cuenca, se utilizó un análisis de correspondencia canónica (ACC), lo que permitió distinguir tres sectores de acuerdo a la altitud. Estos sectores se diferencian en el tipo de sustrato, cobertura riparia vegetal y parámetros físico-químicos, lo que propicia diferencias en la composición de especies de diatomeas entre ellos. Se obtuvieron los valores óptimos calculados y tolerancia calculada para cada una de las especies. Los resultados obtenidos indican que las especies: A. exiguum, $R$. sinuata, L. ventricosa y $C$. tumida, dominan en aguas limpias, oxigenadas y baja temperatura. Las especies $N$. praecipua, $S$. goulardi, C. placentula, L. goeppertiana, N. clausii y $N$. symmetrica se encuentran en aguas cálidas, alta turbidez y poco oxigenadas. Las especies $A$. exiguum, $R$. sinuata, prevalecen en sitios con agua alcalina y baja conductividad y las especies $N$. symmetrica, C. tumida y L. ventricosa presentan una relación positiva con la conductividad y el $\mathrm{pH}$ de las aguas. Las especies estudiadas mostraron buenos indicios de su valor como indicadores de calidad del agua, sin embargo, debido a diferencias de interpretación por varios autores, es necesario realizar más estudios en otras cuencas hidrográficas en el país.

Palabras clave: Costa Rica, diatomeas, ecología, fisicoquímica del agua, Río Sarapiquí, tolerancias, valores óptimos.

\section{REFERENCIAS}

APHA. (2005). Standard methods for the examination of water and wastewater. $21^{\text {st }}$. Ed. American Public Health Association, American Water Works Association, Water Environment Federation: Baltimore, Maryland, USA. 
Azim, M. E., Beveridge, M. C. M., van Dam, A. A., \& Verdegem, M. C. J. (2005). Periphyton and aquatic production: an introduction. In M. E. Azim, M. Beveridge, A. A. van Dam, \& M. Verdegem (Eds.), Periphyton: ecology, exploitation and management (pp. 1-14).Oxfordshire, United Kingdom: CAB International.

Bere, T., \& Tundisi, J. G. (2010). Epipsammic diatoms in streams influenced by urban pollution, Sao Carlos, SP, Brazil. Brazilian Journal of Biology, 70, 921-930.

Bere, T., \& Tundisi, J. G. (2011a.) Influence of land-use patterns in benthic diatom communities and water quality in the trophical Monjolinho hydrological basin, Sao Carlos- SP, Brazil. Water SA, 37, 93-102.

Bere, T., \& Tundisi, J. G. (2011b). Diatom-based water quality assessment in streams influence by urban pollution: effects of natural and two selected artificial substrates, São Carlos-sp, Brazil. Brazilian Journal of Aquatic Science and Technology, 15, 54-63.

Céspedes-Vargas, E. (2014). Análisis taxonómico de las principales especies de diatomeas (Bacillariophyceae) en seis sitios en la Cuenca del Río Sarapiquí, Heredia, Costa Rica (Tesis de Licenciatura). Universidad de Costa Rica, Costa Rica.

Cox, E. J. (1991). What is the basis for using diatoms as monitors of river quality? In B. A. Whitton, E. Rott, \& G. Friedrich (Eds.), Use of algae for monitoring rivers (pp. 33-40). Austria: Institut für Botanik, Universitat Innsbruck.

Goldsborough, L. G. (1994). Heterogeneous Spatial Distribution of Periphytic Diatoms on Vertical Artificial Substrata. Journal of the North American Benthological Society, 13, 223-236.

Hammer, Ø. Harper, D. A. T., \& Ryan, P. D. (2001). PAST: Paleontological statistics software package for education and data analysis. Palaeontologia Electronica, 4(1). Retrived from http://palaeo-electronica. org/2001_1/past/issue1_01.htm

Hasle, G. R., \& Fryxell, G. A. (1970). Diatoms: Cleaning and Mounting for Light and Electron Microscopy. Transactions of the American Microscopical Society, 89, 469-474.

Hynes, H. B. N. (1970). The ecology of running waters. University of Toronto: Toronto, Canada.

Instituto Meteorológico Nacional, Costa Rica (IMN). (2009). Atlas climatológico de Costa Rica, versión digital. IMN: San José, Costa Rica. Retrived from http://www.imn.ac.cr/mapa_clima/interactivo/index. html

Krammer, K., \& Lange-Bertalot, H. (1986). Bacillariophyceae 1. Teil, Naviculaceae. In H. Ettl, J. Gerloff, H. Heynig, \& D. Mollenhauer (Eds.), Süßwasserflora von Mitteleuropa 2 (1) (pp. 887).
Krammer, K. \& Lange-Bertalot, H. (1988). Bacillariophyceae 2. Teil, Bacillariaceae, Epithemiaceae, Surirellaceae. In H. Ettl, J. Gerloff, H. Heynig, \& D. Mollenhauer (Eds.), Süßwasserflora von Mitteleuropa. 2(2). New York, USA: Gustav Fischer Verlag: Stuttgart.

Krammer, K., \& Lange-Bertalot, H. (1991). Bacillariophyceae 3. Teil, Centrales, Fragilariaceae, Eunotiaceae. In H. Ettl, J. Gerloff, H. Heynig, \& D. Mollenhauer (Eds.), Süßwasserflora von Mitteleuropa, 2(3). New York, USA: Gustav Fischer Verlag: Stuttgart.

Krammer, K. (2002). The genus Cymbella. Diatoms of the European Inland Waters and Comparable. Habitats, 3, 1-584.

Lee, R. E. (2008). Phycology. Cambridge: Cambridge, United Kingdom.

Legendre, P., \& Legendre, L. (1998). Numerical Ecology, 2nd English ed. Amsterdam, The Netherlands: Elsevier.

Leopold, L. B., Wolman, M. G., \& Miller, J. P. (Eds.). (1992). Fluvial processes in geomorphology. New York: Dover.

Levkov, Z., \& Ector, L. (2010). A comparative study of Reimeria species (Bacillariophyceae). Nova Hedwigia, 90, 469-489.

Leps, J., \& Smilauer, P. (2003). Multivariate analisis of Ecological Data Using CANOCO. Cambridge, United Kingdom: Cambridge.

Lobo, E., Wetzel, C., Ector, L., Katoh, K., Blanco, S., \& Mayama, S. (2010). Response of epilithic diatom communities to environmental gradients in subtropical temperate Brazilian rivers. Limnetica, 29, 323-340.

Lowe, R. L., \& La Liberté, G. D., (2006). Benthic stream algae: distribution and structure. In F. R. Hauer, \& G. A. Lamberti (Eds.), Methods in stream ecology. Burlington, MA.: American Press.

Metzeltin, D., \& Lange-Bertalot, H. (1998). Tropical Diatoms of South America I. Iconographia Diatomologica. 5. Königstein, Germany: Koeltz.

Metzeltin, D., \& Lange-Bertalot, H. (2007). Tropical Diatoms of South America II. Iconographia Diatomologica. 18. Königstein, Germany: Koeltz.

Michels, A. (1996). Untersuchungen zur Diatomeenvegetation tropischer Fließgewässer und ihre Entwicklung unter dem Einfluß von Abwässern in Costa Rica, Mittelamerika. (Thesis). Universidad Albert-LudwigsFreiburg, Germany.

Michels, A. (1998a). Use of diatoms (Bacillariophyceae) for water quality assessment in two tropical streams in Costa Rica. Revista de Biología Tropical, 46, 153-175. 
Michels, A. (1998b). Effects of sewage water on diatoms (Bacillariophyceae) and water quality in two tropical streams in Costa Rica. Revista de Biología Tropical, 46, 143-152.

Michels, A., Umaña, G., \& Raede, U. (2006). Epilithic diatom assemblages in rivers draining into Golfo Dulce (Costa Rica) and their relationship to water chemistry, habitat characteristics and land use. Archiv für Hydrobiologie, 165, 167-190.

Michels-Estrada, A. (2003). Ökologie und Verbreitung von Kieselalgen in Fliessgewässern Costa Ricas als Grundlage für eine biologische Gewässerbeurteilung in den Tropen. (Dissertationes). Stuttgart, Germany: Cramer.

Morales, E. A., Vis, M. L., Fernández, E., \& Kociolek, J. P. (2007). Epilithic diatoms (Bacillariophyta) from cloud forest and alpine streams in Bolivia, South America II: A preliminary report on the diatoms from Sorata, Department of La Paz. Acta Nova, 3, 680-696.

Morales, E., Luc, E., Fernández, M., Novais, H., Hlúbiková, D., Hamilton, P. B., ... Kociolek, J. P. (2011). The genus Achnanthidium Kütz. (Achnanthales, Bacillariophyceae) in Bolivian streams: a report of taxa found in recent investigations. Algological Studies, 136/137, 89-130.

Nunn, A. (1999). Charakterisierung zweier tropischer Fließgewässer Costa Ricas anhand der Diatomeenund der Makroinvertebratengesellschaften sowie ausgewählter Umweltparameter (Thesis). Estación Limnologica TU-München, Iffeldorf, Germany.

O’Driscoll, C., de Eyto, E., Rodgers, M.,'Connora, M., Asama, Z., \& Xiao, L. (2012). Diatom assemblages and their associated environmental factors in upland peat forest rivers. Ecological Indicators, 18, 443-451.

Phillips, R. E. (1987). Estudio de las poblaciones de diatomeas perifiticas (Bacillariophyceae) en tres riachuelos no contaminados de la Vertiente Atlántica de Costa Rica: Estación Biológica La Selva (OET) (10 24-26'N, 84 00-02'W), Puerto Viejo de Sarapiquí, Heredia (Tesis de Licenciatura). Universidad de Costa Rica, Costa Rica.

Ponader, K., \& Potapova, M. G. (2007). Diatoms from the genus Achnanthidium in flowing waters of the Appalachian Mountains (North America): Ecology, distribution and taxonomic notes. Limnologica, 37, 227-241.

Potapova, M., \& Charles, D. (2007). Diatoms metrics for monitoring eutrophication in rivers of the United States. Ecological Indicators, 7, 48-70.
Roldán-Pérez, G., \& Ramírez-Restrepo, J. J. (2010). Fundamentos de Limnología Neotropical. Colombia: Universidad de Antioquía.

Sala, S., Ramírez, J., \& Plata, Y. (2008). Diatoms from lentic and lotic systems in Antioquia, Chocó and Santander Departments in Colombia. Revista de Biología Tropical, 56, 1159-1178.

Silva, M. (1994). Algal periphyton in two rivers in Costa Rica with especial reference to diatoms organic pollution and altitudinal differences (Doctoral Thesis). Universidad Innsbruck, Austria.

Silva-Benavides, M. (1996a). The epilithic diatom flora of a pristine and a polluted river in Costa Rica, Central America. Diatom. Research, 11, 105-142.

Silva-Benavides, M. (1996b). The use of water chemistry and benthic diatom communities for qualification of a polluted river in Costa Rica. Revista de Biología Tropical, 44, 395-416.

Silva-Benavides, M., Sili, C., \& Torzillo, G. (2008). Cyanoprocaryota y microalgas (Chorophyceae y Bacillariophyceae) bentónicas dominantes en ríos de Costa Rica. Revista de Biología Tropical, 56, 221-235.

Schneck, F., Torgan, L. C., \& Schwarzbold, A. (2007). Epilithic diatom community in a high altitude stream impacted by fish farming in southern Brazil. Study area Epilithic diatom sampling. Acta Limnologica Brasiliensia, 19, 341-355.

Tudesque, L., Grenouillet, G., Gevrey, M., Khazraie, K., \& Brosse, S. (2012). Influence of small-scale gold mining on French Guiana streams: Are diatom assemblages valid disturbance sensors? Ecological Indicators, 14, 100-106.

Veselá, J., \& Johansen, J. R. (2009). The Diatom Flora of Ephemeral Headwater Streams in the Elbsandsteingebirge Region of the Czech Republic. Diatom Researcher, 24, 443-477.

Vouilloud, A., Sala, S., Nunez, A., \& Duque, S. (2010). Diatoms from the Colombian and Peruvian Amazon: the genera Encyonema, Encyonopsis and Gomphonema (Cymbellales: Bacillariophyceae). Revista de Biología Tropical, 58, 45-62.

Williams, D. M. (1986). Comparative morphology of some species of Synedra Ehrenb. with a new definition of the genus. Diatom Research, 1, 131-152.

Wydrzycka, U., \& Lange-Bertalot, H. (2001). Las diatomeas (Bacillarioyphyceae) acidófilas del Río Agrio y sitios vinculados con su cuenca, Volcán Poás, Costa Rica. Brenesia, 55/56, 1-68. 


\section{APÉNDICE 1}

Área de muestreo en la sección media de la cuenca del Río Sarapiquí, Costa Rica.

\section{APPENDIX 1}

Sample area in the middle reaches of the drainage basin of Sarapiquí River, Costa Rica.

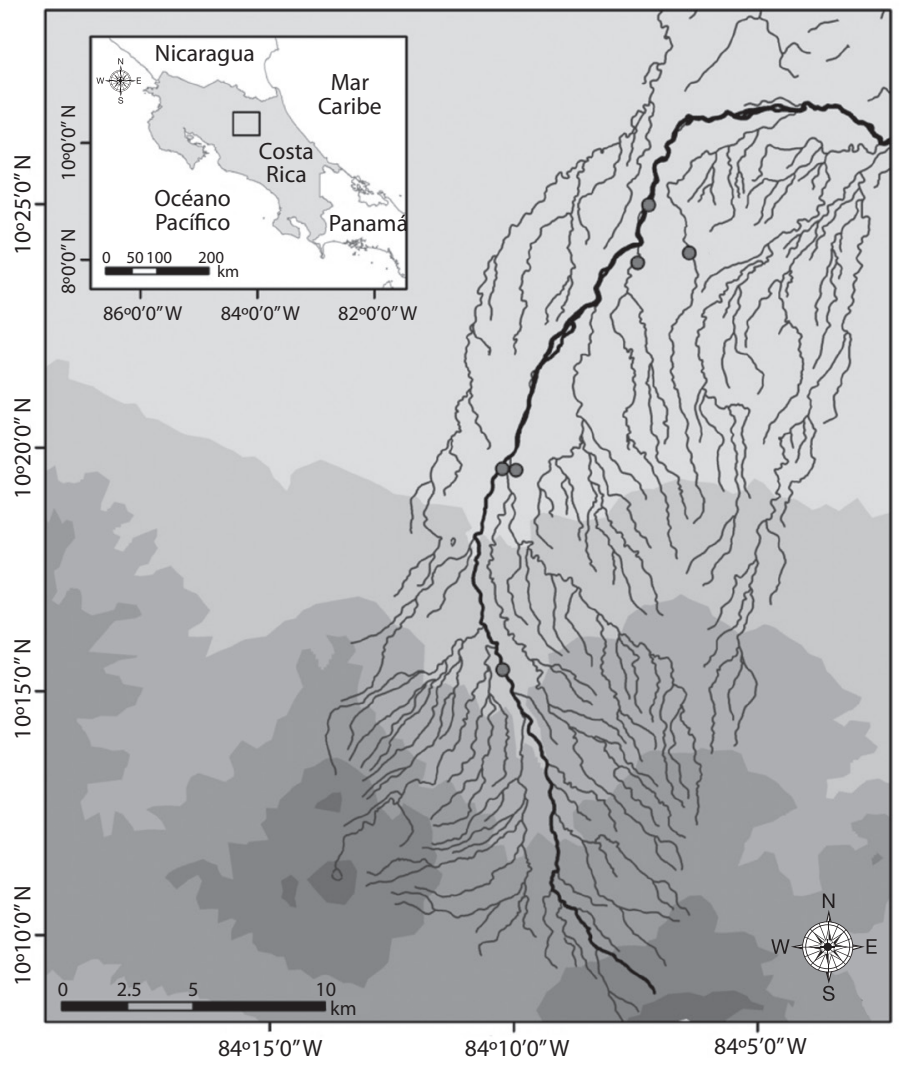

Elevación $(\mathrm{m})$

$0-500$

$501-1000$

$1001-1500$

$1501-2000$

$2001-2500$

$2501-3000$

— Río Sarapiquí, cauce principal Afluentes

- Sitio de muestreo

1. Sar 1: Sarapiquí, Colonia Socorro 2. Sar 2: Sarapiquí, San Miguel

3. Sar 3: Sarapiquí, Reserva la Tirimbina

4. Vol: Río Volcán

5. S. Ram: Río San Ramón

6. Tiri: Río Tirimbina 
